\title{
Novel Participatory Methodologies for Identifying Motivations of Minority Women to Participate in a Breast Cancer Clinical Trial
}

\author{
Madeline Evans ${ }^{1}$, Katherine E. Ridley-Merriweather ${ }^{2}$ \\ ${ }^{1}$ Indiana University School of Medicine; ${ }^{2}$ Susan G. Komen Tissue Bank at the IU Simon Cancer \\ Center
}

Background: The Komen Tissue Bank (KTB), a biorepository that stores healthy breast tissue for use as controls in breast cancer $(B C)$ research, has a notable deficiency in tissue donations from racial and ethnic minority women. Though studies have documented similar disparities in many clinical trials, the reasons why minority women agree or decline to participate in clinical trials remain largely unknown. This study used two novel participatory communication methodologies with minimal researcher intervention to explore potential motivations for minority women (Latinas, Asian Americans, and Black women) to donate breast tissue to the KTB.

Methods: Two novel methodologies rooted in Grounded Practical Theory and Grounded Theory were used to study the attitudes of non-donors toward tissue donation and the reasons donors chose to donate tissue to the KTB, respectively. The non-donors (Latinas, $n=14$; Asian Americans, $n=17$ ) participated in interactive focus groups, and donors (Black, $n=20$ ) participated in open-ended interviews, all of which were then transcribed and coded to identify common motivations for donating breast tissue.

Results: The interactive methodology of both the focus groups and interviews yielded rich data that may not have arisen in a traditional question-driven format. Though the manifestation differed between minority groups, three common factors influencing the decision to donate breast tissue were present in all groups: altruistic tendencies, cultural norms, and personal connection to BC. Non-donors also expressed their need for more information before making the decision to donate.

Conclusion and Potential Impact: Common factors that influence the decision to donate tissue were identified using these two novel interactive methodologies, though the expression differed between minority groups. The information gleaned from this study will inform future communication efforts by the KTB and other clinical trial researchers, with the ultimate goal of distributing targeted recruitment materials to increase the representation of minority women in clinical trials. 